\title{
Scleral Lens and Prosthetic Replacement of the Ocular Surface Ecosystem Utilization in Ocular Graft-versus-Host Disease: A Survey Study
}

\author{
Shannon M Bligdon' \\ Bradley A Colarusso ${ }^{2}$ \\ Allen Y Ganjei $\mathbb{D}^{3}$ \\ Alan Kwok ${ }^{4}$ \\ Zhonghui Katie Luo ${ }^{2}$ \\ Daniel Brocks (D) ${ }^{4}$ \\ 'Ajax Eye Care, Ajax, ON, Canada; \\ ${ }^{2}$ Massachusetts Eye and Ear, Boston, MA, \\ USA; ${ }^{3}$ Drexel University College of \\ Medicine, Philadelphia, PA, USA; \\ ${ }^{4}$ BostonSight, Needham, MA, USA
}

Correspondence: Alan Kwok

BostonSight, 464 Hillside Ave, Unit 205,

Needham, MA, 02494, USA

Tel + | 78| 7267337

Fax + I 78I 7267310

Email akwok@bostonsight.org
Purpose: To evaluate the utilization of scleral lenses and prosthetic replacement of the ocular surface ecosystem devices (SL/PDs) in the management of ocular graft-versus-host disease (oGVHD)

Patients and Methods: A survey of 15 questions was sent via email to 6032 subjects registered with the Blood and Marrow Transplant Information Network. The survey reviewed transplant history, graft-versus-host disease history, as well as oGVHD symptoms and onset. Additional questions surveyed treatments used for oGVHD, as well as the degree of ocular symptom control and experience with SL/PDs. A total of 306 respondents met the eligibility requirements to be part of the analyzed cohort.

Results: The mean number of symptoms reported from the analyzed cohort was $4.79 \pm 2.44$, median (IQR) of 5.0 (3.0 to 7.0), with the most common symptom being gritty, dry eyes $(87 \%)$. The mean number of treatments utilized across the analyzed cohort was $3.21 \pm 2.55$, median (IQR) of 2.5 (1.0 to 5.0), with the most common treatment being artificial tears $(86 \%)$. Wearing scleral lenses resulted in a mean of $5.42 \pm 1.86$, median (IQR) of 6.0 (4.0 to 7.0) symptoms improving, with improved dryness/grittiness of the eyes (94\%), improved eye pain $(92 \%)$ and improved quality of life $(89 \%)$ being the most commonly improved symptoms. Fifty-six percent of those wearing scleral lenses wished the lenses had been recommended sooner. The most common reason patients cited for not wearing scleral lenses was that they had never heard of them $(63 \%)$.

Conclusion: SL/PDs help to control the symptoms of oGVHD. With their use, clinicians are able to improve the quality of life of this patient population. Despite the known benefits, SL/ PDs still remain underutilized in oGVHD care. A majority of current SL/PD wearers wish that they had been recommended sooner as a treatment option. SL/PDs should be considered a component of comprehensive oGVHD management.

Keywords: GVHD, oGVHD, dry eye, keratoconjunctivitis sicca, QOL, HSCT, allo-HSCT

\section{Introduction}

Graft-versus-host disease (GVHD) is a multifactorial immune mediated response characterized by transplanted donor $\mathrm{T}$ cells attacking host antigens. It occurs following allogeneic hematopoietic stem cell transplantation (allo-HSCT) where stem cells are transplanted from one unidentical individual to another using stem cells harvested from bone marrow, peripheral blood or umbilical cord. Allo-HSCT is used to treat both benign and malignant hematological conditions, including certain leukemias, lymphomas and non-dysplastic syndromes. There is a lack of consensus on the incidence rate of GVHD following allo-HSCT, with reports 
ranging from $25-70 \% .{ }^{1,2}$ The skin, liver, and oral mucosa are most commonly involved; however, the gastrointestinal tract, lungs, esophagus, and eyes can also frequently be affected, with a reported ocular graft-versus-host disease (oGVHD) incidence of $40-60 \%$ following allo-HSCT. ${ }^{3,4}$

The chief clinical manifestation of oGVHD is keratoconjunctivitis sicca with concurrent meibomian gland dysfunction and meibomian gland atrophy. ${ }^{5}$ This leads to corneo-conjunctival inflammation which can result in epithelial erosions, filamentary keratitis and the most severe clinical manifestations of corneal stromal ulceration, melt and perforation. ${ }^{6,7}$ Patients will often experience significant pain, debilitating visual impairment and a profound decrease in their quality of life (QOL) due to the intractable nature of the disease ${ }^{8-10}$ For patients with chronic systemic GVHD the reported incidence of oGVHD varies, with some projections as high as 60$90 \% .^{11}$

Scleral lenses and prosthetic replacement of the ocular surface ecosystem devices (SL/PDs), (PROSE, BostonSight, Needham, MA) are a standardized, FDAapproved therapeutic treatment modality indicated for a variety of ocular surface diseases including oGVHD. ${ }^{12}$ Both prospective and retrospective studies report their use and benefit in the management of the signs and symptoms of the disease. ${ }^{3,12}$ SL/PDs are large diameter, rigid gas permeable lenses that vault over the corneal surface and rest on the sclera/conjunctiva. They reduce surface inflammation and provide epithelium support. Constant lubrication is provided to the corneal surface by way of a fluid reservoir. The rigidity of the lens material protects the ocular surface from the sheering forces of the eyelids and the large diameter of the lens provides defense from environmental factors (air/wind, pollutants). With the introduction of these lenses in the oGVHD population, patients can find significant relief. ${ }^{13,14}$

Historically, the use of SL/PDs in oGVHD was reserved for only severe forms of the disease. In the modern era we have seen an increase in the accessibility of SL/PDs, improvement in lens design and technology and a reduction in cost to both the provider and the patient. This, along with standardized optometric training and extensive efficacy data available in peer-reviewed journals, has made SL/PDs appropriate in a wide range of ocular surface disease states ranging from mild and moderate to severe. However, there continues to be a persistent misconception among clinicians that the use of this treatment option remains only for severe disease. Anecdotally, oGVHD patients regularly report to SL/PD providers that this misconception has resulted in delayed referrals for SL/ PD fitting following years of attempted use of other treatment modalities. The underutilization in mild, moderate and severe oGVHD has seemingly resulted in an unnecessary prolonged reduction in QOL for patients prior to referral. The goal of this survey was to evaluate and more definitively ascertain practice patterns and the utilization of SL/PDs for the management of oGVHD.

\section{Materials and Methods}

\section{Study Design}

The authors created a 15-question survey to characterize patient GVHD and oGVHD history, time course, ocular symptoms, prior treatments, and history of SL/PD use (Appendix 1). Demographic information, including age and gender, was recorded. The first 7 survey questions directly addressed transplant history, GVHD history, and characterized oGVHD symptoms and their onset. Subsequent questions addressed past treatment modalities used, rating the current level of ocular symptom control, and assessing any prior or current use of SL/PDs. The final questions, when applicable, addressed in more detail each subject's experience with SL/PDs, including characterizing symptomatic response. Throughout the survey, question logic was employed to guide a subject through an appropriate question pathway given their personal experience with oGVHD, ocular symptoms and treatments.

\section{Data Collection}

Via electronic mail, a link to the study survey (SurveyMonkey, San Mateo, CA) was sent to 6032 blood and bone marrow transplant patients who were members of the Blood and Marrow Transplant Information Network (BMTinfonet https://www.bmtinfonet.org), a not-for-profit 501(c) 3 transplant advocacy organization. Subjects were first emailed the survey through BMTInfonet on January 17th, 2021. Those who did not respond received a second email requesting participation on January 24th, 2021. Subjects who completed the survey were entered into a draw for a $\$ 200$ gift card prize.

\section{Statistical Analysis}

Statistical analysis was performed using Microsoft Excel and the Statistical Package for the Social Sciences (SPSS) program version 21.0 (IBM Corp., Armonk, New York, NY, USA) 
unless otherwise noted. Normality was determined utilizing an online Shapiro-Wilk Test calculator (https://www.statsking dom.com/shapiro-wilk-test-calculator.html). Continuous normally distributed variables are represented as mean \pm standard deviation. Continuous variables with non-normal distribution are reported with mean \pm standard deviation along with median (interquartile range [IQR]). Comparison of data sets with confirmed normality are analyzed via the applicable $T$-test (paired $T$-test or two sample $T$-test [Welch's]). Nominal and ordinal categorical variables were examined for relationships utilizing the chi-square test. Significance was set to a P-value of $\leq 0.05$ in all instances of analysis.

\section{Ethical Approval}

The study was reviewed and determined to be exempt from IRB review under category \#2(ii) as detailed in 45 CFR 46.104(d) and BRANY's standard operating procedure, by the BRANY IRB (an independent institutional review board) on 1/19/2021, BRANY IRB File \# 21-12-019-784. All procedures and activities were performed in accordance with relevant state and local law and followed strict ethical obligations as set forth by the Declaration of Helsinki and Good Clinical Practice (GCP). HIPPA requirements do not apply because use of or access to $\mathrm{PHI}$ is not required for this research and the data collected did not include PHI.

\section{Results}

Three hundred seventy-one subjects with a history of alloHSCT responded to the 6032 emails sent, resulting in a response rate of $6.15 \%$. Three hundred thirty-nine of these subjects indicated they had ocular symptoms, however, 4 of them did not complete the questionnaire and therefore were excluded from the analyzed cohort. Participants with ocular symptoms were then asked to characterize the onset of their symptoms in relation to their transplant, which identified 306 subjects whose symptoms began after their transplant, suggestive of carrying the diagnosis of oGVHD. Of the 306 subjects, $260(85 \%)$ reported a formal diagnosis of GVHD anywhere in their body, with 170 of the 306 subjects $(56 \%)$ reporting a formal diagnosis of oGVHD by their physician. Results for the responses of these 306 subjects are reported below.

\section{Demographics}

In terms of gender, 193 respondents $(63 \%)$ were female and $113(37 \%)$ were male. The average age was $59.6 \pm$ 12.2, median (IQR) of 62 (55 to 68 ) years.

\section{Symptoms}

The variety of presumed oGVHD symptoms exhibited within the study population is shown in Figure 1. The mean number of symptoms was $4.79 \pm 2.44$, median (IQR) of 5.0 (3.0 to 7.0) out of a total of 9 symptom options. The most common symptoms were gritty, dry eyes $(87 \%)$, light sensitivity $(61 \%)$, burning or stinging sensations $(59 \%)$, and eye pain $(52 \%)$.

When questioned more specifically about symptom onset, for 107 subjects (35\%) their eye symptoms started within 6 months after their transplant, while for 87 subjects $(28 \%)$ their eye symptoms started 7-12 months after their transplant. For 112 subjects (37\%), their eye symptoms started more than 1 year after their transplant.

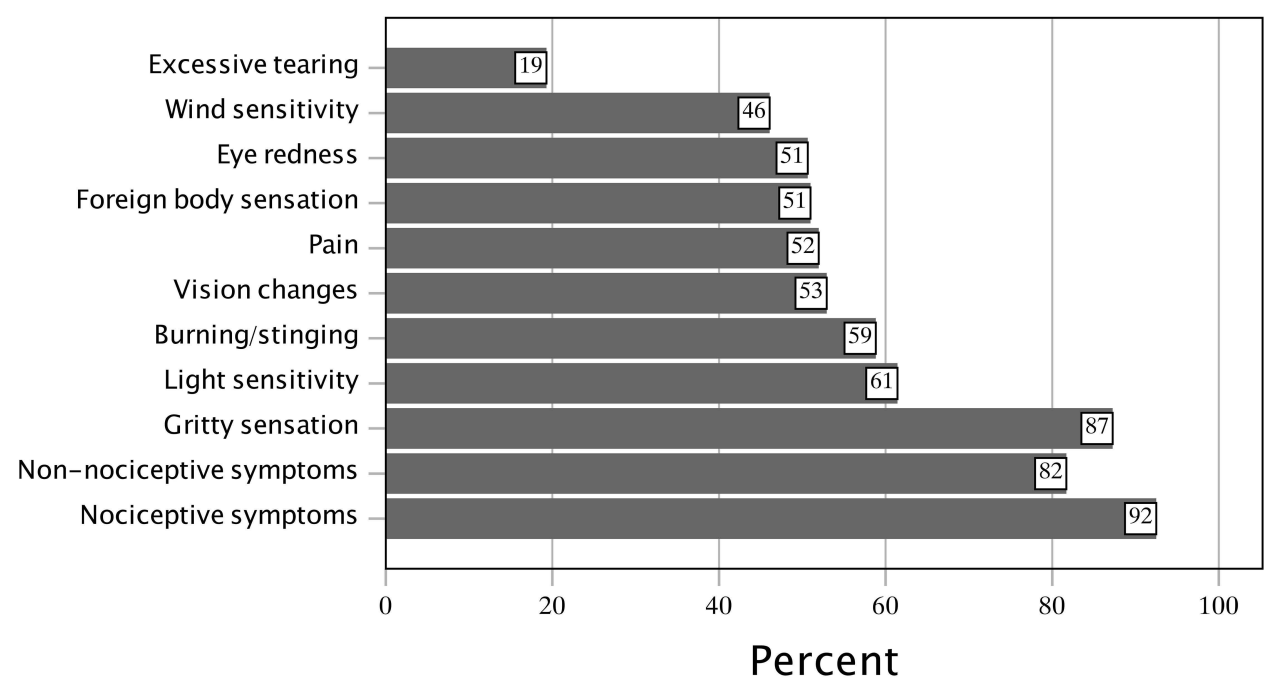

Figure I Reported symptoms in subjects with presumed ocular graft-versus-host disease. 
Table I Subject Self-Reported Symptom Control Rating

\begin{tabular}{|l|c|c|c|}
\hline & $\begin{array}{c}\text { Current } \\
\text { SL/PD } \\
\text { Wearer }\end{array}$ & $\begin{array}{c}\text { Prior SL/ } \\
\text { PD } \\
\text { Wearer }\end{array}$ & $\begin{array}{c}\text { Never SLI } \\
\text { PD } \\
\text { Wearer }\end{array}$ \\
\hline $\begin{array}{l}\text { Well-controlled } \\
\%(n) \\
\text { Moderately- } \\
\text { controlled } \\
\%(n) \\
\begin{array}{l}\text { Poorly-controlled } \\
\%(n)\end{array}\end{array}$ & $25.00(9)$ & $6.25(1)$ & $33.93(76)$ \\
\hline Total \% (n) & $5.56(2)$ & $31.25(5)$ & $100.71(24)$ \\
\hline
\end{tabular}

Abbreviation: SL/PD, scleral lens/prosthetic replacement of the ocular surface ecosystem device.

When asked to rate their current eye symptoms, 86 participants $(28 \%)$ described their symptoms as wellcontrolled, 159 (52\%) described their symptoms as moderately-controlled, and $31(10 \%)$ described their symptoms as poorly-controlled. Thirty subjects $(10 \%)$ did not rate their symptoms, and these patients were excluded from the remaining analysis. Self-reported symptom control categorized by SL/PD wearing status is presented in Table 1 .

\section{Treatments}

Treatments utilized for the relief of oGVHD symptoms are described in Figure 2. The mean number of treatments was $3.21 \pm 2.55$, median (IQR) of 2.5 (1.0 to 5.0). The most common treatments for oGVHD symptoms included artificial tear drops (86\%), lubricating ointments (52\%), Restasis or Cequa (cyclosporine ophthalmic emulsion $0.05 \%$ or $0.09 \%)(42 \%)$, and steroid eye drops $(36 \%)$.

\section{SL/PD Use}

In the studied population, 36 subjects $(13 \%)$ were actively wearing an SL/PD, 16 subjects $(6 \%)$ had previously worn an SL/PD but had stopped, and 224 subjects (81\%) had never worn an SL/PD. Seventy-seven subjects (25\%) stated that SL/PD had been suggested as a possible treatment option by their physician. Of these 77 respondents, 25 (32\%) had never worn an SL/PD. Of those who were actively wearing an SL/PD, all $(100 \%)$ had been prescribed lenses after their transplant.

There was no significant association between gender and current $(\mathrm{P}=0.226)$ or past $(\mathrm{P}=0.629) \mathrm{SL} / \mathrm{PD}$ use. There was also no association between gender and never having used an SL/PD $(\mathrm{P}=0.732)$.

There was no significant association between age and SL/ PD referral $(60.19 \pm 9.51$ years with referral vs $59.52 \pm 13.12$ years without referral; $\mathrm{P}=0.636$ ). There was no association between subject age and current $(\mathrm{P}=0.901)$ or past $(\mathrm{P}=0.825)$ $\mathrm{SL} / \mathrm{PD}$ use, or never having used an SL/PD $(\mathrm{P}=0.730)$.

Subjects who actively use an SL/PD had a history of being more symptomatic from their ocular surface disease than subjects who did not currently use one; $7.28 \pm 1.26$, median (IQR) of 8.0 (7.0 to 8.0) total symptoms vs $4.46 \pm$ 2.37, median (IQR) of 4.0 (2.25 to 6.75) total symptoms (P $<0.001)$. Subjects who have never used an SL/PD had a history of significantly fewer total number of ocular symptoms than those who had used or currently use one $(4.52 \pm 2.25$ vs $5.55 \pm 2.79 ; \mathrm{P}=0.019)$.

When comparing current users versus those who have never used an SL/PD, current wearers have tried more treatments than patients who have never worn an SL/PD

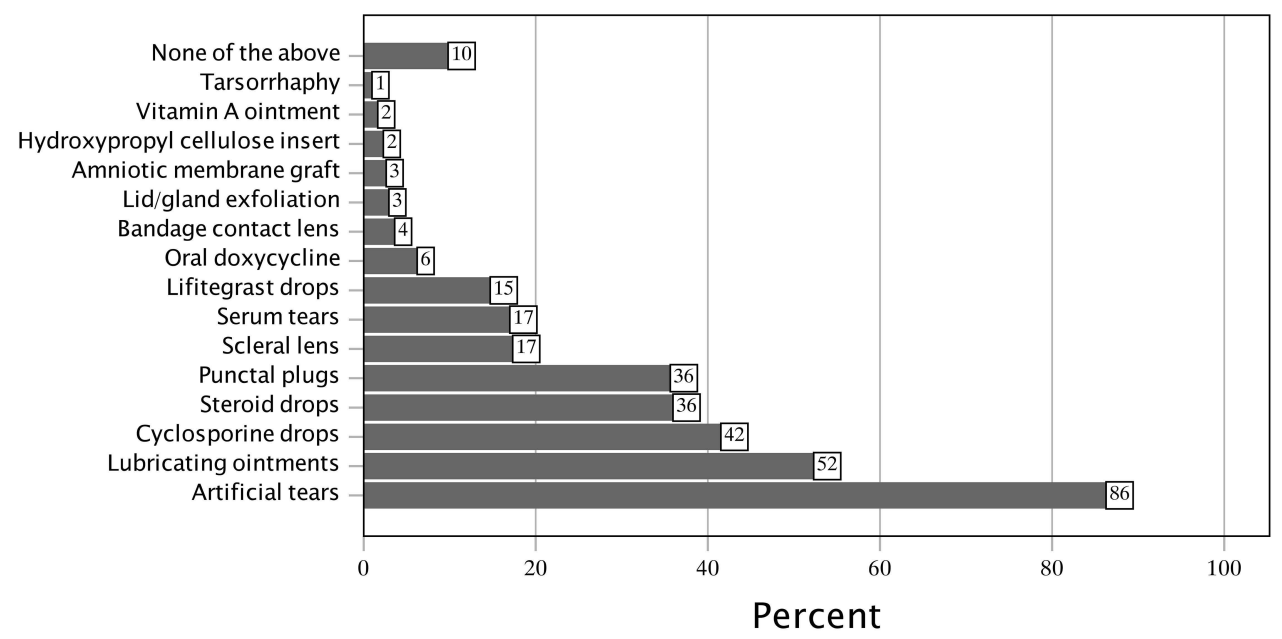

Figure 2 Treatments utilized by subjects with presumed ocular graft-versus-host disease. 


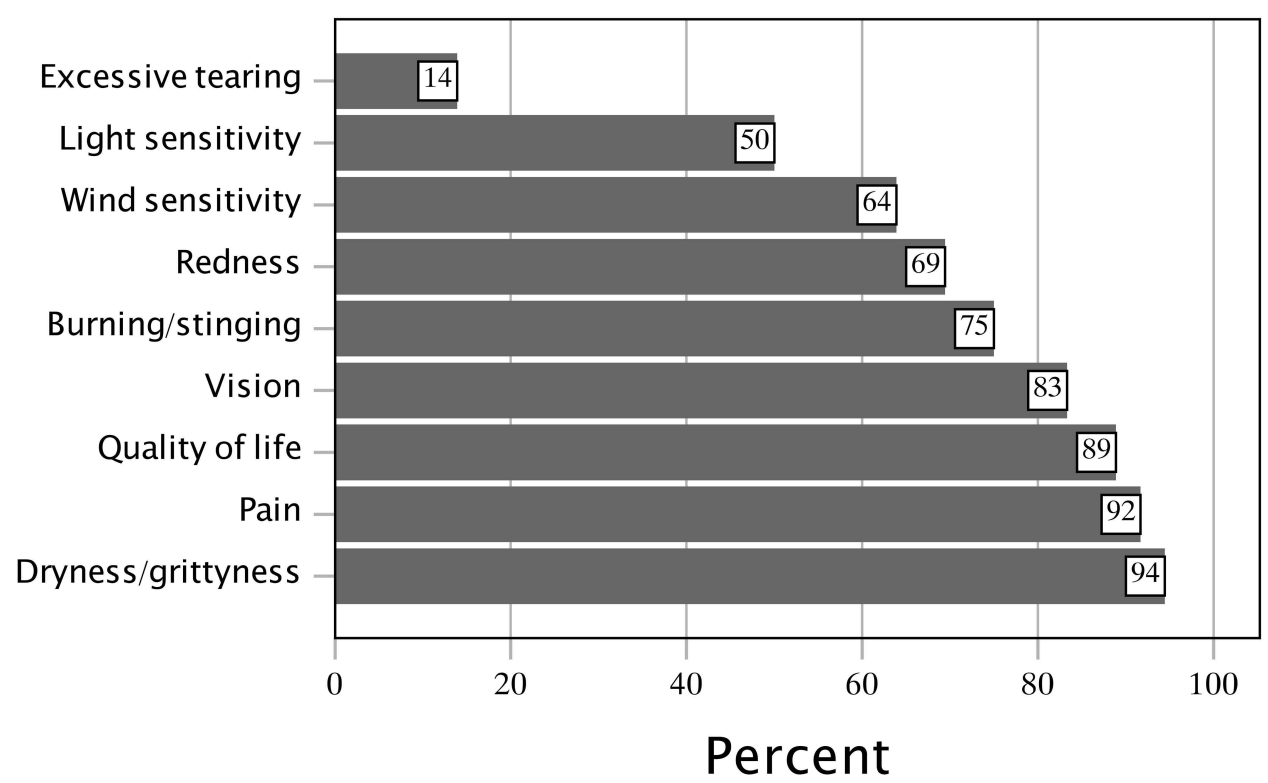

Figure 3 Symptoms improved as a result of SL/PD use in current SL/PD wearers.

Abbreviation: SL/PD, scleral lens/prosthetic replacement of the ocular surface ecosystem devices.

and this observation was statistically significant; $6.42 \pm$ 1.50, median (IQR) of 6.5 (5.75 to 7.0$)$ vs $2.78 \pm 1.93$, median (IQR) of $2.0(1.0$ to 4.0$)(\mathrm{P}<0.001)$.

\section{Improvements}

Subjects who currently wear an SL/PD were questioned about what improvements occurred in their oGVHD symptoms as a result of wearing it (Figure 3). The mean number of improved symptoms resulting from SL/PD wear was 5.42 \pm 1.86 , median (IQR) of 6.0 (4.0 to 7.0). Most common were improvements in the dryness or grittiness of their eyes (94\%), improved eye pain (92\%), improved overall quality of life (89\%), and improved vision (83\%). Twenty subjects (56\%) currently wearing an SL/PD wished they had been recommended an SL/PD sooner in their disease course.

\section{Discontinuation}

Subjects who had discontinued their SL/PD use described why they had stopped (Figure 4). The mean number of reasons for discontinuation was $3.56 \pm 1.79$, median (IQR) of 3.0 (2.75 to 5.0). The most common reasons for discontinuation were difficulty handling the lens (69\%), fogging or mucus build-up (69\%), and discomfort (50\%).

\section{SL/PD Never Used}

We asked subjects who had never used an SL/PD why they had not done so. Of our subjects, 141 (63\%) had never heard of an SL/PD while 53 (24\%) had heard of SL/PDs but were never referred; 4 (2\%) were referred for an SL/PD but never attended an appointment, and $6(2 \%)$ were fitted for lenses but never used them. Twenty subjects $(9 \%)$ reported "other" as the reason for never having worn an SL/PD.

\section{Discussion}

Patients with oGVHD will often experience a diminished QOL as a result of their ocular symptoms, which most commonly include burning, pain, visual blur and light sensitivity. $9,15,16$ These symptoms can negatively impact an individual's ability to perform tasks of daily living, socialize and even preclude them from leaving their house due to severe ocular pain. ${ }^{10}$ With the utilization of SL/PDs, eye care providers are able to dampen or eliminate these symptoms and allow patients to lead happier and more fulfilled lives. ${ }^{17}$

Our survey found that, of the patients currently wearing an SL/PD, the top three reported benefits of lens wear were improved dryness, improved eye pain, and improved QOL (94\%, 92\% and 89\%, respectively). Of the 9 symptoms surveyed, an average improvement of 5.42 symptoms was reported to have resulted from SL/PD wear by current SL/PD wearers, illustrating the efficacy of these lenses/ devices for oGVHD management. This is in agreement with previous studies on the use of SL/PD in the management of the signs and symptoms of oGVHD. In a study of 79 oGVHD eyes, Theophanous et al observed a corneal staining improvement in $84 \%$ of eyes and a visual acuity improvement in $90 \%$ of eyes from their pre-fitting 


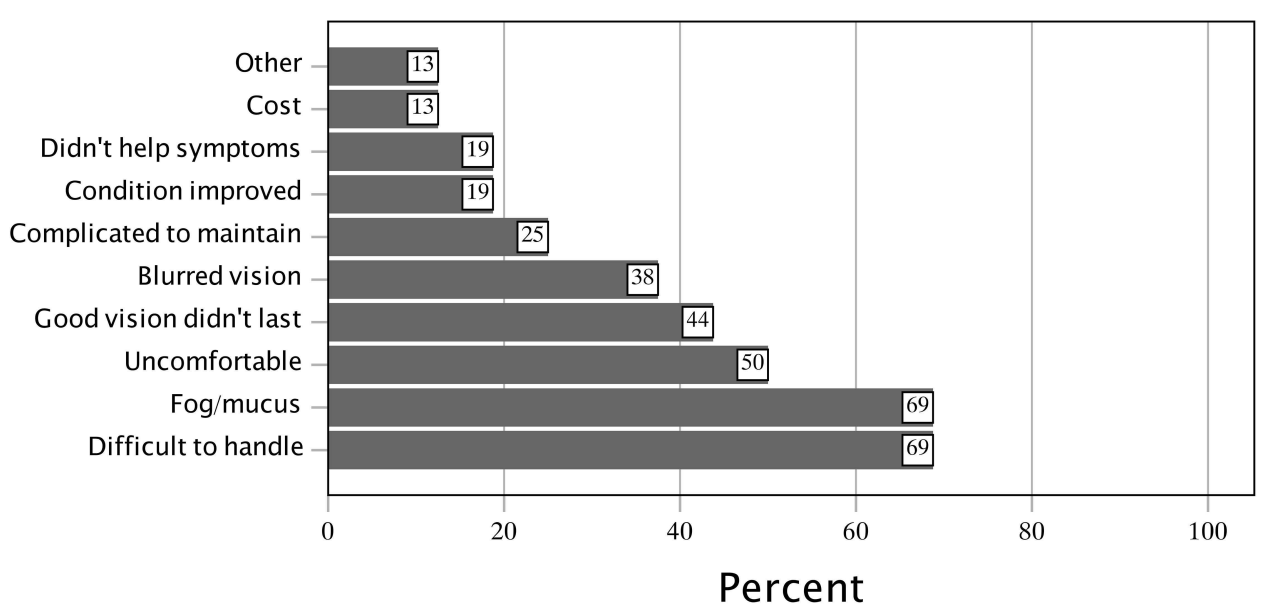

Figure 4 Reasons for discontinuation of SL/PD in former users.

Abbreviation: SL/PD, scleral lens/prosthetic replacement of the ocular surface ecosystem devices.

consultation following daily use of prosthetic replacement of the ocular surface ecosystem devices. ${ }^{3}$ Jacobs and Rosenthal, in a study of 33 patients wearing prosthetic replacement of the ocular surface ecosystem devices, found that $73 \%$ of respondents reported a "great improvement" in QOL, with improvements in activities such as driving (92\%) and reading (93\%). ${ }^{17}$ In 2007, Takahide et al reported that the use of scleral lenses helped promote corneal healing and helped patients to resume their normal life activities. ${ }^{12}$

SL/PDs, however, are not without their challenges. In our survey, for the subgroup of subjects who started SL/ PD wear but subsequently ceased wearing lenses, the most common reasons for discontinuation were fogging/mucus build up (69\%), difficulty in handling, such as with insertion and removal (69\%), and lenses being uncomfortable (50\%). SL/PDs are known to have more robust handling regimens and disinfection requirements compared to other soft and rigid lens modalities, making them some of the most common reasons for scleral lens dropout. ${ }^{18}$ oGVHD patients also often experience increased mucus production, which can deposit on the front surface of their SL/PD, causing visual blur, thus requiring frequent removal and re-application. ${ }^{9}$ To combat this commonly encountered challenge, SL/PD wearers may utilize a variety of surface wetting techniques, including the frequent application of preservative-free artificial tears, sweeping a lens removal plunger or cotton swab moistened with a rigid lens conditioning solution over the front of the lens whilst on the eye, or removing, rinsing and reinserting their lenses as needed. In more extreme cases of heavy surface deposition, a low dose topical steroid applied to the naked eye may be warranted.

At present, SL/PD use in the management of oGVHD is not considered first line therapy by eye care professionals. This is made evident by the fact that only $12 \%$ of our survey respondents reported they actively wear an SL/PD. The most common treatments reported included artificial tears $(86 \%)$, lubricating ointments $(52 \%)$, and topical cyclosporine eye drops $(42 \%)$. These more commonly reported treatments are consistent with the current tiered management recommendations for dry eye disease by the Tear Film Ocular Society Dry Eye Workshop (DEWS) II in 2017. ${ }^{19}$ This report categorized artificial lubricants as a step one treatment, topical cyclosporine as a step two treatment, followed by scleral lenses and surgical intervention in steps three and four, respectively. ${ }^{19}$

The primary purpose of oGVHD treatment is to increase lubrication, reduce tear evaporation, decrease surface inflammation, and provide epithelial support. ${ }^{20} \mathrm{SL} /$ PDs, by design, can help achieve each of these therapeutic goals concomitantly and yet clinician utilization of them for the oGVHD patient population is still limited. This underutilization is multifactorial, likely biased by historical precedent and particularly centered around previous access and financial barriers. To fit and dispense prosthetic replacement of the ocular surface ecosystem devices requires certification through specific hours of virtual and in-person training, which can be a limiting factor to utilization expansion. Scleral lenses, manufactured by commercial labs, do not have this restriction, which has been one attribute that has fostered clinician adoption. Our survey found that patients wearing an SL/PD had 
a history of significantly more symptoms than patients who do not wear a lens/device. Those wearing an SL/PD have also tried more treatments than those who do not. Those individuals who reported never having worn an SL/ PD rated their symptoms as "well-controlled" more frequently (34\%) than current (25\%) and previous (6\%) SL/ PD users. Taken in toto, these results highlight the continued concentration of SL/PD use in those who are more severely affected and at more advanced stages of the disease. It is important to note, in cases of mild oGVHD, that SL/PD technology may not be required or found to be of significant benefit, particularly for those whose symptoms are adequately controlled through more conservative, first-line therapies. However, intervening sooner for the cohort that has never worn SL/PDs could result in an improvement in symptomatic control, specifically for the $66 \%$ in this group not currently self-reporting "wellcontrolled" symptoms. The need for consideration of earlier interventions is evident; $56 \%$ of survey participants who were current SL/PD wearers wished they had been recommended sooner.

Recent advancements in lens design, technology, manufacturing, and standardized clinician training have resulted in increased access to this treatment modality and a reduced financial burden. With ever-growing options for customization, there continues to be headway for improved lens tolerance, decreased fit challenges and even greater success of this modality in the oGVHD population. Decreasing the burden on providers and patients in the fitting and wearing of SL/PDs encourages the implementation of this treatment earlier in the disease process, and thus can increase quality of life and reduce the financial and emotional hardship resulting from years of other procedures and treatments.

Despite the well-reported and accepted benefits of SL/PD wear in oGVHD, suboptimal patient and physician education of this treatment option continues to be a barrier to utilization. $^{13,21}$ Only a quarter of our survey respondents reported that an SL/PD had ever been suggested to them as a management option. For those who reported that they had never used a scleral lens, $63 \%$ reported that they had never even heard of SL/PDs and 24\% reported having heard of SL/ PDs but never being referred for a fitting. Reasons for this disconnect could include a lack of understanding by GVHD providers (oncologists, oncology medical support staff and nursing, ophthalmologists, optometrists) of the advantages of SL/PDs, leading to a failure to disseminate information and educate oGVHD patients. Clinical care models may reserve referring patients for SL/PD treatment to only those with more advanced symptoms or after they have failed using more traditional measures. As is seen in other dry eye diseases, in some cases patient signs in oGVHD can be disproportionately severe compared to clinically lesser symptoms. This may lead to a delay in referral for those clinicians predominantly focused on symptoms as the trigger for SL/ PD referral, further articulating the importance of knowledgeable and experienced oGVHD providers. ${ }^{22,23}$

Demographics may also play a vital role in utilization. Access to SL/PD care, though improved, is often still geographically limited, especially in more rural areas. The financial burden of SL/PD, particularly due to a lack of medical insurance coverage in many instances, is an ongoing barrier. Medical insurance providers often fail to differentiate between routine contact lens daily wear for vision (such as soft contact lenses) versus medically necessary scleral lenses for the treatment of sight-threatening pathology such as oGVHD.

The design of this survey is subject to limitations. Our survey population was derived from the Blood and Marrow Transplant Information Network (BMTinfonet), an advocacy organization that provides information and support to transplant patients. This may produce a sampling bias from a more educated cohort, as patients who subscribed to BMTinfonet are regularly provided with GVHD educational resources. A more educated patient population may be more knowledgeable about treatment options, may more proactively search out treatment options, and may bias data toward increased utilization of available treatment resources. Future survey studies including multiple patient outreach modalities (multiple online support groups, social media, in-office questionnaires in locations throughout the US and internationally) may provide an avenue for more broad patient population participation. Out of 6032 surveys emailed to BMTinfonet subscribers, 371 responses were received, for a response rate of $6.15 \%$. While this level of response is modest, and may be considered a limitation, we must bear in mind who makes up the population receiving the email requesting participation in the survey. Not all recipients carried the diagnoses of GVHD or oGVHD and therefore may have self-selected non-participation.

Additionally, a survey study comes with inherent potential weaknesses, particularly in the risk of recall bias. The subjective nature of patient-recalled data, including oGVHD diagnosis, history, symptoms, and treatments, may confound our analysis. Recall bias may particularly be at risk when comparing those with severe disease who may spend quite significant time and energy recalling and 
reviewing their long-suffering journey compared to those with mild disease.

Another consideration is that other dry eye etiologies may have contributed to our respondents' symptoms, thereby misidentifying them as oGVHD. We attempted to control for this by only including the 306 respondents who reported onset of ocular surface disease symptoms following their transplant, making the diagnosis of oGVHD most likely. This definition for inclusion in the analyzed cohort seemed reasonable and purposely erred on the side of inclusion, as only including those 170 respondents who reported a formal diagnosis of oGVHD would be overly exclusionary for a population that is recognized as being underdiagnosed. ${ }^{24}$ However, we must recognize that these reported subjects with presumed oGVHD most accurately could be termed subjects with "ocular surface disease symptoms following allo-HSCT" given the lack of objective information available from the survey. The diagnosis of oGVHD does formally necessitate objective clinical manifestations via ophthalmic slit lamp evaluation and testing of the ocular surface and tear film integrity. However, even with inoffice examination, given the wide range of signs and symptoms, this formal diagnosis can often be overlooked and is still subject to clinician bias. ${ }^{20}$ In our study, the lack of objective data assessing and confirming oGVHD diagnosis, signs and severity, and response to treatment produces a limitation inherent to patient-recall survey studies. Future prospective survey studies could include initial objective confirmation of oGVHD as a formal diagnosis prior to allowing participation in the survey portion of the study. ${ }^{25}$

This study did not record or report on serious adverse events relatable to any mentioned treatments including scleral lenses. For those that discontinued scleral lens wear, some of the reasons for discontinuation, including lens fogging/mucus build up, fluctuating vision and blurred vision, could be considered adverse events but are more appropriately categorized as intolerances. However, 9\% of subjects who discontinued scleral lenses chose the option "other" as their reason for doing so and it is certainly possible that an adverse event such as infectious keratitis could have played a role, but this specific information was not recorded. Prior studies have described the risks of scleral lens wear, including the risk of infectious keratitis, in oGVHD patients as well as other subgroups. ${ }^{26-28}$ Additional studies, particularly those prospective in nature, could more appropriately and more accurately collect such information and provide this data to the clinician to use as an important part of the calculation of when to utilize scleral lenses.

Lastly, our study had relatively few patients who currently use or have previously used SL/PDs $(12 \%$ and $5 \%$, respectively) compared to patients who have never used an SL/PD (73\%), which could skew statistical analysis given unequal cohorts. There were also more female $(63 \%)$ than male (37\%) respondents, which is in line with oGVHD predilection for female gender. However, it has been shown that women with dry eye disease will often report an increased severity of symptoms, greater use of treatments, and lower treatment satisfaction compared to men. ${ }^{29,30}$ This may create a response bias in our survey questions pertaining to symptom control and treatments. Despite this propensity found in females, there was no significant association between gender and SL/PD use in our survey population.

Future studies should include a randomized clinical trial to examine a larger cohort of SL/PD wearers with varying disease severity to further understand the breadth of symptom control and treatment efficacy. Additional investigation is also needed to evaluate current GVHD provider management strategies and referral metrics for utilizing SL/PDs. Ongoing studies will ultimately allow for an updated optimization of SL/PD preferred practice patterns for the treatment of oGVHD.

\section{Conclusion}

The survey results indicate that additional education regarding SL/PD utilization for the treatment of oGVHD is needed for eye care and tertiary care providers as well as for oGVHD patients. This will be even more important as allo-HSCT becomes increasingly prevalent in treating benign and malignant hematological diseases thereby collaterally expanding the number of people suffering from oGVHD. By implementing SL/PD early as part of a multifaceted oGVHD treatment regimen, practitioners have the ability to decrease patient morbidity and improve QOL. GVHD and oGVHD patients should be routinely referred to an eye care professional with an ocular surface disease specialty and SL/PD knowledge in order to ensure that comprehensive evaluation and management occurs.

\section{Data Sharing Statement}

The data that support the findings of this study are available from the author, AG, upon reasonable request. The authors confirm that the data supporting the findings of this study are available within the article. 


\section{Acknowledgments}

The authors acknowledge the support of BMTInfonet (bmtinfonet.org) in disseminating the survey.

\section{Funding}

This research received no specific grant from any funding agency in the public, commercial, or not-for-profit sectors. It was supported by the authors' sundry funds and other institutional resources.

\section{Disclosure}

A. Kwok and D. Brocks are salaried employees of BostonSight, Needham, MA, where BostonSight PROSE treatment and BostonSight SCLERAL lenses were developed. None of the authors have a propriety interest in PROSE treatment, the prosthetic devices, any commercially available scleral lenses or any relevant financial disclosures. The authors report no other conflicts of interest in this work.

\section{References}

1. Munir SZ, Aylward J. A review of ocular graft-versus-host disease. Optom Vis Sci. 2017;94(5):545-555. doi:10.1097/OPX.0000000000001071

2. Nassiri N, Eslani M, Panahi N, Mehravaran S, Ziaei A, Djalilian AR. Ocular graft versus host disease following allogeneic stem cell transplantation: a review of current knowledge and recommendations. J Ophthalmic Vis Res. 2013;8:351-358.

3. Theophanous C, Irvine JA, Parker P, Chiu GB. Use of prosthetic replacement of the ocular surface ecosystem scleral lenses in patients with ocular chronic graft-versus-host disease. Biol Blood Marrow Transplant. 2015;21:2180-2184. doi:10.1016/j.bbmt.2015.07.027

4. Espana EM, Shah S, Santhiago MR, Singh AD. Graft versus host disease: clinical evaluation, diagnosis and management. Graefes Arch Clin Exp Ophthalmol. 2013;251:1257-1266. doi:10.1007/s00417-013-2301-z

5. Ogawa Y, Kuwana M. Dry eye as a major complication associated with chronic graft-versus-host disease after hematopoietic stem cell transplantation. Cornea. 2003;22(Supplement 1):S19-S27. doi:10.10 97/00003226-200310001-00004

6. Inamoto Y, Valdes-Sanz N, Ogawa Y, et al. Ocular graft-versus-host disease after hematopoietic cell transplantation: expert review from the late effects and quality of life working committee of the center for international blood and marrow transplant research and transplant complications working party of the European society of blood and marrow transplantation. Biol Blood Marrow Transplant. 2019;25: e46-e54. doi:10.1016/j.bbmt.2018.11.021

7. Nassar A, Tabbara KF, Aljurf M. Ocular manifestations of graft-versus-host disease. Saudi J Ophthalmol. 2013;27(3):215-222. doi:10.1016/j.sjopt.2013.06.007

8. Jacobs DS, Carrasquillo KG, Cottrell PD, et al. CLEAR - medical use of contact lenses. Cont Lens Anterior Eye. 2021;44(2):289-329. doi:10.1016/j.clae.2021.02.002

9. Chiu GB, Theophanous C, Irvine JA. PROSE treatment in atypical ocular graft-versus-host disease. Optom Vis Sci. 2016;93 (11):1444-1448. doi:10.1097/OPX.0000000000001003

10. Riemens A, Te Boome LC, Kalinina Ayuso V, et al. Impact of ocular graft-versus-host disease on visual quality of life in patients after allogeneic stem cell transplantation: questionnaire study. Acta Ophthalmol. 2014;92:82-87. doi:10.1111/aos.12047
11. Na KS, Yoo YS, Mok JW, Lee JW, Joo CK. Incidence and risk factors for ocular GVHD after allogeneic hematopoietic stem cell transplantation. Bone Marrow Transplant. 2015;50:1459-1464. doi:10.1038/bmt.2015.187

12. Takahide K, Parker PM, Wu M, et al. Use of fluid-ventilated, gas-permeable scleral lens for management of severe keratoconjunctivitis sicca secondary to chronic graft-versus-host disease. Biol Blood Marrow Transplant. 2007;13:1016-1021. doi:10.1016/j. bbmt.2007.05.006

13. DeLoss KS, Le HG, Gire A, Chiu GB, Jacobs DS, Carrasquillo KG. PROSE treatment for ocular chronic graft-versus-host disease as a clinical network expands. Eye Contact Lens. 2016;42:262-266. doi:10.1097/ICL.0000000000000186

14. Romero-Rangel T, Stavrou P, Cotter J, Rosenthal P, Baltatzis S, Foster CS. Gas-permeable scleral contact lens therapy in ocular surface disease. Am J Ophthalmol. 2000;130:25-32. doi:10.1016/S00029394(00)00378-0

15. Saboo US, Amparo F, Abud TB, Schaumberg DA, Dana R. Vision-related quality of life in patients with ocular graft-versus-host disease. Ophthalmology. 2015;122(8):1669-1674. doi:10.1016/j.ophtha.20 15.04.011

16. Dietrich-Ntoukas T, Cursiefen C, Westekemper H, et al. Diagnosis and treatment of ocular chronic graft-versus-host disease: report from the German-Austrian-Swiss consensus conference on clinical practice in chronic GVHD. Cornea. 2012;31:299-310. doi:10.1097/ ICO.0b013e318226bf 97

17. Jacobs DS, Rosenthal P. Boston scleral lens prosthetic device for treatment of severe dry eye in chronic graft-versus-host disease. Cornea. 2007;26 (10):1195-1199. doi:10.1097/ICO.0b013e318155743d

18. Barnett M, Courey C, Fadel D, et al. CLEAR - scleral lenses. Cont Lens Anterior Eye. 2021;44:270-288. doi:10.1016/j.clae.2021.02.001

19. Jones L, Downie LE, Korb D, et al. TFOS DEWS II management and therapy report. Ocul Surf. 2017;15:575-628. doi:10.1016/j. jtos.2017.05.006

20. Nair S, Vanathi M, Mukhija R, Tandon R, Jain S, Ogawa Y. Update on ocular graft-versus-host disease. Indian J Ophthalmol. 2021;69:1038-1050. doi:10.4103/ijo.IJO_2016_20

21. Schornack MM, Baratz KH, Patel SV, Maguire LJ. Jupiter scleral lenses in the management of chronic graft versus host disease. Eye Contact Lens. 2008;34:302-305. doi:10.1097/ ICL.0b013e318188e205

22. Vehof J, Sillevis Smitt-Kamminga N, Nibourg SA, Hammond CJ. Predictors of discordance between symptoms and signs in dry eye disease. Ophthalmology. 2017;124:280-286. doi:10.1016/j. ophtha.2016.11.008

23. Begley CG, Chalmers RL, Abetz L, et al. The relationship between habitual patient-reported symptoms and clinical signs among patients with dry eye of varying severity. Invest Ophthalmol Vis Sci. 2003;44:4753-4761. doi:10.1167/iovs.03-0270

24. Moyal L, Adam R, Akesbi J, Rodallec FT, Nordmann JP. [Ocular graft-versus-host disease: an often misdiagnosed etiology of dry eye syndrome]. J Fr Ophtalmol. 2017;40:122-125. French. doi:10.1016/j. jfo.2016.08.012

25. Espandar L, Moshirfar M, Kim SK. Diagnosis and Management of Ocular Graft Vs. Host Disease. EyeNet; 2021.

26. Stoyanova EI, Otten HM, Wisse R, Rothova A, Riemens A. Bandage and scleral contact lenses for ocular graft-versus-host disease after allogeneic haematopoietic stem cell transplantation. Acta Ophthalmol. 2015;93(7):e604. doi:10.1111/aos.12711

27. Jeong M, Lee KL, She RC, Chiu GB. Microbiological evaluation of opened saline bottles for scleral lens use and hygiene habits of scleral lens patients. Optom Vis Sci. 2021;98:250-257. doi:10.1097/ OPX.0000000000001657

28. Fuller DG, Wang Y. Safety and efficacy of scleral lenses for keratoconus. Optom Vis Sci. 2020;97(9):25-32. doi:10.1097/ OPX.0000000000001578 
29. Pellegrini M, Bernabei F, Barbato F, et al. Incidence, risk factors and complications of ocular graft-versus-host disease following hematopoietic stem cell transplantation. Am J Ophthalmol. 2021;227:25-34. doi:10.1016/j.ajo.2021.02.022
30. Schaumberg DA, Uchino M, Christen WG, Semba RD, Buring JE, Li JZ. Patient reported differences in dry eye disease between men and women: impact, management, and patient satisfaction. PLoS One. 2013;8(9):e76121. doi:10.1371/journal.pone.0076121

\section{Publish your work in this journal}

Clinical Ophthalmology is an international, peer-reviewed journal covering all subspecialties within ophthalmology. Key topics include: Optometry; Visual science; Pharmacology and drug therapy in eye diseases; Basic Sciences; Primary and Secondary eye care; Patient Safety and Quality of Care Improvements. This journal is indexed on PubMed

Submit your manuscript here: https://www.dovepress.com/clinical-ophthalmology-journal
Central and CAS, and is the official journal of The Society of Clinical Ophthalmology (SCO). The manuscript management system is completely online and includes a very quick and fair peer-review system, which is all easy to use. Visit http://www.dovepress.com/ testimonials.php to read real quotes from published authors. 